\title{
Significance of buried object orientation variation in their detection using thermography
}

\author{
by V. Krstelj, M. Malinovec, J. Stepanić Jr. and S. Švaić \\ University of Zagreb, Faculty of Mechanical Engineering and Naval Architecture, \\ I. Lučića 5, HR-10000 Zagreb, Croatia, T: ++38516168 222, F: ++38516156940
}

\begin{abstract}
Landmines are part of a complex system with variable characteristics that may change with time. If thermography is applied on such a system, the most significant characteristics of resulting thermograms is overcritical noise which has severely suppressed the application of thermography for landmine detection for humanitarian demining. The attempts to realise as much as possible of the thermography potential motivated concentrating onto sources of noise in buried objects thermograms, in particular onto variations in mine orientation relative to the soil normal. The experiments reported in this paper were performed in order to investigate the influence of angle between the local vertical axis and mine symmetry axis, the angle between the local vertical axis and soil surface normal, and the object depth. The influences are quantified and ranked through the statistically planned experiment. The strongest influence is the statistical interaction of depth and angles. According to the statistical test these two combinations are significant influences. The results indicate that the application of modern thermography in humanitarian demining is to be broadened by including the variations in mine orientation.
\end{abstract}

\section{Introduction}

Humanitarian demining and related technologies [1] are not efficient enough regarding the quantity of mined areas to be cleared [2]. Its part connected with most of duration and risk is mine detection [3]. One of the approaches in R\&D work conducted in order to overcome this problem is the application of thermography based landmine detection which offers the possibility of fast and non-contact detection, hence it is less risky and less time consuming than techniques used nowadays. However, at the present level of development, thermography is concentrated mainly on soil surface $[4,5]$ and thin soil subsurface layer detection, e.g. of object depths below $5 \mathrm{~mm}$ [6] or equal to $8 \mathrm{~mm}$ [7], which is all rather far from requirements imposed by UN for landmine removal of at least $130 \mathrm{~mm}$ depth [8] (Figure 1).

Two main groups of factors that limit the depth sensitivity of thermography are (i) variations and non-homogeneities of mine-neighbouring soil [9 - 12], and (ii) the diffusive character of the thermal response from the buried object which is intrinsically linked to heat conduction in the soil [6]. Their influence is generally seen in randomly distributed changes of the projections of buried object boundaries and in the suppression of the thermal contrast caused by the local difference in thermal characteristics, respectively. These limitations are known for some time and caused the mentioned concentration of R\&D work onto surface laid landmines and detection of shallowly buried landmines. Hence the detection of deeply buried landmines was dealt with rather exceptionally $[9,11$, 12], which is the reason why important pieces of information regarding buried landmines conditions in realistic mine affected regions are missing. Processes influencing the state of landmine-soil system, which bring about the listed two groups of factors, and which are relevant in thermography based detection, can be divided into two groups. In the first group (group A) are processes which locally modify thermal parameters and heat conduction in a generally unknown way. In the literature the influence of water content and other environmental factors was analysed $[11,12]$. These include non-stationary and non- 
homogeneous water transfer through the soil and soil-air boundary, vegetation growth and ceasing, influence of animals, etc. Due to these influences landmines are not shown on thermograms as objects of regular shape. In the second group (group B) are processes affecting the prevalently expected position of a landmine parallel to the soil surface. Therefore landmines seemingly may have a more complex shape than horizontal landmines. In case of landmines, soil erosion brings about their lateral [13] and vertical [14] translations, as well as the possibility of changes in the angle between their axis and the local vertical axis [15]. All this results in a potentially broad spectrum of landmine orientations.

Group B is important because the regular shape of a buried landmine allows to extract its signature from a variety of thermal signatures originating from objects of irregular shape. For example, if neural networks are used for thermogram evaluation, the network will probably not detect a non-vertical landmine by matching its shape with extrapolated circular, almost circular, and rectangular shapes of landmines used in the phase of network learning. Additionally, while thermal characteristics of the local soil can be evaluated in preliminary, usually rather fast measurements, the orientations of existing landmines cannot be found in advance. Generally, the probability of detection and the related reliability level depend on the landmine-soil states, which differ in landmines orientation. However, on the basis of available literature, one can argue that this influence has not been adequately studied.

In order to evaluate the relevant consequences of conditions in which landmines are found, the emphasis of this work is put on the different types of variations in the landminesoil system, i.e. on the parameters belonging to group B processes. This way we contribute to the analysis of different sources of thermogram noise. Using statistical planning of experiments [16] we obtained for each of them the quantitative expression of significance, together with the significance of their combinations. In the interpretation, the influences of parameters $\theta$ and $\psi$ were compared to the influence of $d$.

The experimental set-up used in this work is described in the next section. Experimental results, in this realisation of active thermography, are given in the third section and are discussed in the fourth section, thereafter conclusions and lines of future development.

\section{Experimental set-up}

Homogeneous steel discs of equal dimensions are used as a representation of buried objects in order to contribute to the repeatability of the experiment $[7,10]$. Because of their axial symmetry, their orientation is described using the angle $\theta$ between their symmetry axis and the vertical direction. A further simplification is that the soil surface is taken as a homogeneous plane. The normal to the soil surface lies in the plane spanned by the vertical direction and the landmine axis, and forms the angle $\psi$ with the vertical axis, Figure 2. In this simplified geometry these two angles are sufficient to characterise landmine orientation. As was shown before, the landmine signature on the thermogram depends on its depth $[6,7,10]$ which is the vertical distance $d$ between the soil and the centre of the upper cylinder base, Figure 2.

In order to quantify the relative influence strength of these parameters on the characteristics of thermograms, a plan according to statistical test $2^{3}$ was formulated [16]. With a given reliability level that test allows to compare the influences of the angles with the influence of the depth and to show the importance of interactions [16], which are five combinations of the three factors compared. Values of parameters in the experiment were $2 \mathrm{~mm}$ and $4 \mathrm{~mm}$ for $d, 0^{\circ}$ and $12^{\circ}$ for $\theta$ and $0^{\circ}$ and $7^{\circ}$ for $\psi$. Intervals of angles were determined based on our previous experience with their variations. All eight combinations of parameters were used in the experiment, with three measurements for each combination. The measurements were taken in random order [16]. 
The experimental set-up for these investigations included a liquid nitrogen cooled thermography camera AGA 680 Standard, a box filled with sand, steel cylinders, and a heater (Figure 3). During the experiment, the surface temperature did not exceed $400 \mathrm{~K}$. Starting from room temperature, the soil above the buried cylinders was heated during 5 min with $6 \mathrm{~kW} \cdot \mathrm{m}^{-2}$ heat intensity. The thermograms with large enough thermal contrast were recorded during the first 3 minutes of cooling.

\section{Results}

The temperature profiles follow the standard form of a relatively fast rise during heating followed by slower cooling, where the time lag increases with depth, like in other similar situations [10].

Using simple procedures, thermograms were transformed into scalar quantity - a boundary roundness $b$ which is calculated numerically from thermograms. During the experiments the temperature difference above the mines was about several tenths of $K$ while the temperature sensitivity was not higher than $0.2 \mathrm{~K}$. Therefore the signature of a buried object consisted only of one or two isotherms of effectively isolated objects. The boundary roundness for one isotherm was formed from the angular distribution of the isotherm radii, measured from its centre. Boundary roundness was then calculated as the ratio of variance of that distribution divided by the squared average value of radius. The form chosen emphasises the deviation of a shape from a circle. The 24 values of $b$ obtained from recorded thermograms (Fig. 4) were inputs for the statistical test $2^{3}$. Its results (Table 1) are relative strengths of isolated and combined effects, i.e. statistical interactions, of parameter changes on $b$, obtained in decomposition of variance of the set of values for $b$.

\section{Discussion}

A general characteristic of active thermography experiments is non-stationarity. Transient heat flux imposed on the soil surface induces formation of local, transient heat sinks or heat sources. In the case considered, cylinders were heat sinks during heating and in the first minutes of cooling. After that, they acted as heat sources. Therefore in the thermograms a buried cylinder was colder than the soil surface around it.

Another general characteristic of the buried object thermography is that the signatures of deeper objects have smoother boundaries. Here, that effect was not strong since the thermal resolution of the camera was low. However, it is seen on thermograms that deeper parts of objects look different from shallower ones. During heating, deeper parts are in a given moment colder than shallower ones. During cooling, heat is transferred from deeper parts to a larger part of a soil than from shallower ones. Hence on thermograms a part of a buried object signature corresponding to its deeper part is smoother and thermally less contrasted. In our experiments the difference in distance between the objects' parts and the soil surface was parameterised by the angles $\theta$ and $\psi$. In the case considered, the thermal resolution limit of the camera suppressed the evidence of such an effect. However, statistically it was possible to extract the effect.

The statistical tests exploited how variations in $d, \theta$ and $\psi$ affect the roundness of observed boundaries. From the results of the experiment (i.e. the values of $b$ ) we evaluated the strength of an isolated effect, or a bilinear or trilinear combination, i.e. statistical interaction. The hypothesis underlying the tests is that the roundness significantly differs from a circle. The critical $F$ test values, with significance levels 0.05 and 0.01 , are 4.49 and 8.53 , respectively. Effects having $F$ test values above the critical one are considered significant, i.e. their influence on roundness is larger than the error in the value of $b$. It is calculated (Table 2) that significant effects (both on the 0.05 and 0.01 level) are combined effects of $d$ and angles $\theta$ and $\psi$. The $\mathrm{F}$ test values of these two effects are similar, indicating that their effect on $b$ is similar. These two significant effects are followed by the three effects where only one of the angular variables $\psi, \theta \psi$ and $\theta$ changes. 
These three effects are of comparable strength, pointing out that they have similar effects on $b$. The combination $d \theta \psi$ is somewhat smaller than linear and bilinear effects involving angles. Finally, the influence of $d$ on the changes of boundary roundness is the smallest one. As this is to be expected its value serves as a contribution to the quality of results.

\section{Conclusions and lines of future development}

It is argued that the three-parameter model represents a significant group of realistic variations of the buried landmine-neighbouring soil systems. Combinations of these parameters are sorted according to their influence on thermograms obtained in active thermography configuration. Using a factorial design of experiment, it was shown that changes in the orientation of a buried object significantly influence the roundness of its signature on the thermograms of the surface above it during transient phase of soil thermal response. The origin of that influence is connected to the local changes of the depths where the parts of the object are buried.

As variations of orientation affect the signature shape displayed by thermograms of buried objects, the thermography techniques should include such variations in order to be applicable in mine detection in humanitarian demining. This is particularly important because, prevalently, it is the regular (circular or rectangular) shape of a buried mine which is to be recognised in thermograms. The results obtained are important for practitioners in the development of thermography systems applicable in humanitarian demining for noncontact mine detection.

In order to obtain the total influence of the angles on the thermogram of the buried object, the maximum values of variations are to be considered. Though such values are relatively rare, their occurrence may reduce the detection probability below the UN requested value [1]. Therefore the results obtained are valid in similar experimental setups, i.e. for metal objects shallowly buried in homogeneous soils without dense vegetation, and for given ranges of parameter variations. However, having in mind the underlying heat conduction, we conjecture that results obtained are valid in much broader classes of situations, in which polymer objects are buried in a broad range of depths and in different soils.

In order to improve the understanding of how the three parameters exploited here act on the different characteristics of thermograms, the denser coverage of three-dimensional parameter space is needed. Considering furthermore that realistic situations are characterized using a larger number of characteristics [17], results of the experiment conducted serve as a starting point for the development of different multi-parameter models that are suited for the detection of buried objects using thermography.

\section{Acknowledgments}

This work was financed through the projects of Croatian Ministry of Science and Technology No. 120098 and No. 120021.

\section{REFERENCES}

[1] UN Mine Action Service - International Mine Action Standards, Ch. 4. - Glossary, available at: http://www.mineactionstandards.org/IMAS_archive/Final/04.10.pdf,

[2] Kingsbury (N.) - Land Mine Detection DOD's Research Program Needs a Comprehensive Evaluation Strategy, US GAO Report, GAO-01 239, 2001,

[3] Krstelj (V.) and Stepanić (J.) - Humanitarian de-mining detection equipment and working group for antipersonnel landmines detection. INSIGHT, Vol. 42(3), 2000, pp. 187-190,

[4] Dubey (A. C.), Harvey (J. F.), Broach (J. T.) and George (V.) eds. - Detection and Remediation Technologies for Mines and Minelike targets VI, Sections EO/IR I - V, SPIE Proceedings Vol. 4394, 2001, 
[5] Merino (O. G.) - Image analysis of Infrared Polarization measurements of landmines, Thesis, Vrije Universiteit Brussel, 2001,

[6] Lundberg (M.) and Gu (I. Y. H.) - A 3-D matched filter for detection of land mines using spatio-temporal thermal modelling, Detection and Remediation Technologies for Mines and Minelike targets V, SPIE Proceedings Vol. 4038, pp. 179-188, 2000,

[7] Boras (I.), Malinovec (M.), Stepanić (J.) and Švaić (S.) - Modelling of Buried Object Detection using Thermography, QIRT 2000, Reims, Proceedings, pp. 176-181, 2002 ,

[8] UN Mine Action Service - International Mine Action Standards, Ch. 9. - Clearance requirements, available at: http://www.mineactionstandards.org/IMAS_archive/Final/ 09.10.pdf,

[9] Pregowski (P.), Swiderski (W.) and Walczak (R.) - Surface and volume effects in thermal signatures of buried mines: experiment and modeling. QIRT '98, Lodz, Proceedings, 1998, pp. 233-238,

[10] Boras (I.), Malinovec (M.), Stepanić (J.) and Švaić (S.) - Detection of Underground Objects using Thermography, $15^{\text {th }}$ WCNDT, Rome, 2000, Proceedings, available at: http://www.ndt.net/article/wcndt00/papers/idn106/idn106.htm,

[11] Baertlein (B. A.) and Sendur (I. K.) - The Role of Environmental Factors and Mine Geometry in Thermal IR Mine Signatures, Detection and Remediation Technologies for Mines and Minelike targets VI, SPIE Proceedings Vol. 4394, pp. 449-460, 2001,

[12] Hong (S.-h.) et al. - Impact of soil water content on landmine detection using radar and thermal infrared sensors, Detection and Remediation Technologies for Mines and Minelike targets VI, SPIE Proceedings Vol. 4394, pp. 409-416, 2001,

[13] Stipčić (R. T.) - Director of the demining company Ru-Ru, private communication,

[14] Molnar (Š.) - Head of the UXO Department - Hungarian Military Forces, private communication,

[15] Gasser (R.) - Technology for Humanitarian Landmine Clearance, Ph.D. Thesis, University of Warwick, 2000,

[16] Mendenhall (W.) and Sincich (T.) - Statistics for the Engineering and Computer Sciences, Dellen Publ. Comp., San Francisco, 1988,

[17] Boras (I.), Krstelj (V.), Malinovec (M.), Stepanić (J.) and Švaić (S.) - Advanced Analysis of Thermograms of Buried Objects in Non-homogeneous Environment, QIRT 2002, Dubrovnik, Proceedings, to be published. 


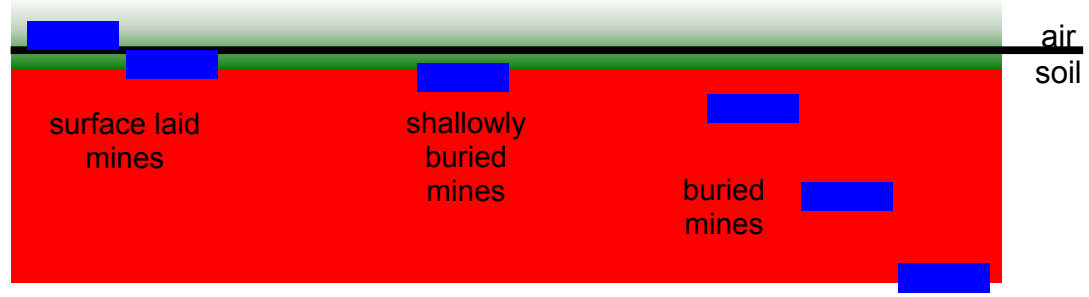

Fig. 1. Comparison of required (green and red) and realised (green) depths of detection of landmines using thermography

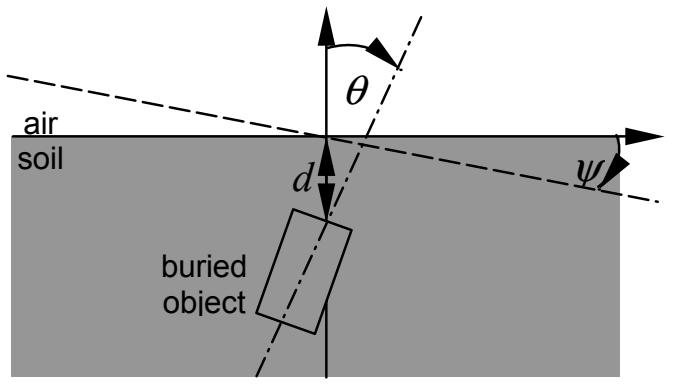

Fig. 2. Extracted parameters

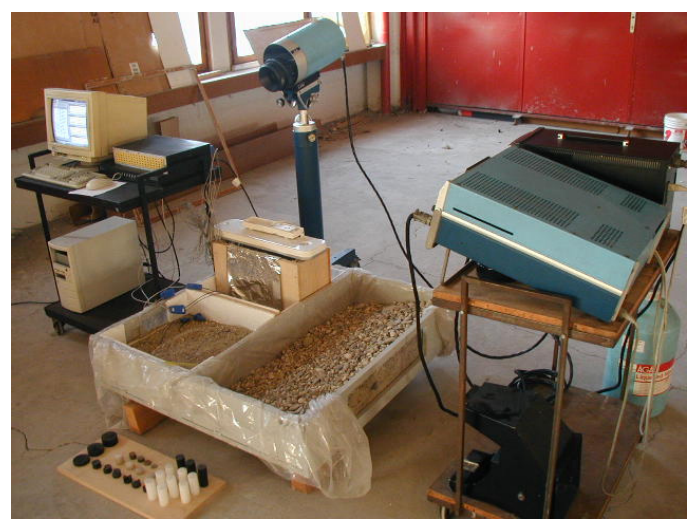

Fig. 3. Experimental set-up

Table 1: Values of relative strengths of isolated and combined parameters. Significant contributions are significant both at the $1 \%$ and $5 \%$ level, as the critical values for the encountered number of degrees of freedom are $\mathrm{F}(0.05)=4.49$ and $\mathrm{F}(0.01)=8.53$.

\begin{tabular}{|c|c|c|c|c|c|c|c|}
\hline Contribution & $\boldsymbol{d}$ & $\boldsymbol{\theta}$ & $\boldsymbol{\psi}$ & $\boldsymbol{d} \boldsymbol{\theta}$ & $\boldsymbol{d} \psi$ & $\boldsymbol{\theta} \boldsymbol{\psi}$ & $\boldsymbol{d} \theta \boldsymbol{\psi}$ \\
\hline$F$ test value & 0.03 & 1.47 & 2.44 & 11.6 & 13.9 & 1.73 & 0.95 \\
\hline Significance & No & No & No & Yes & Yes & No & No \\
\hline
\end{tabular}


http://dx.doi.org/10.21611/qirt.2002.009

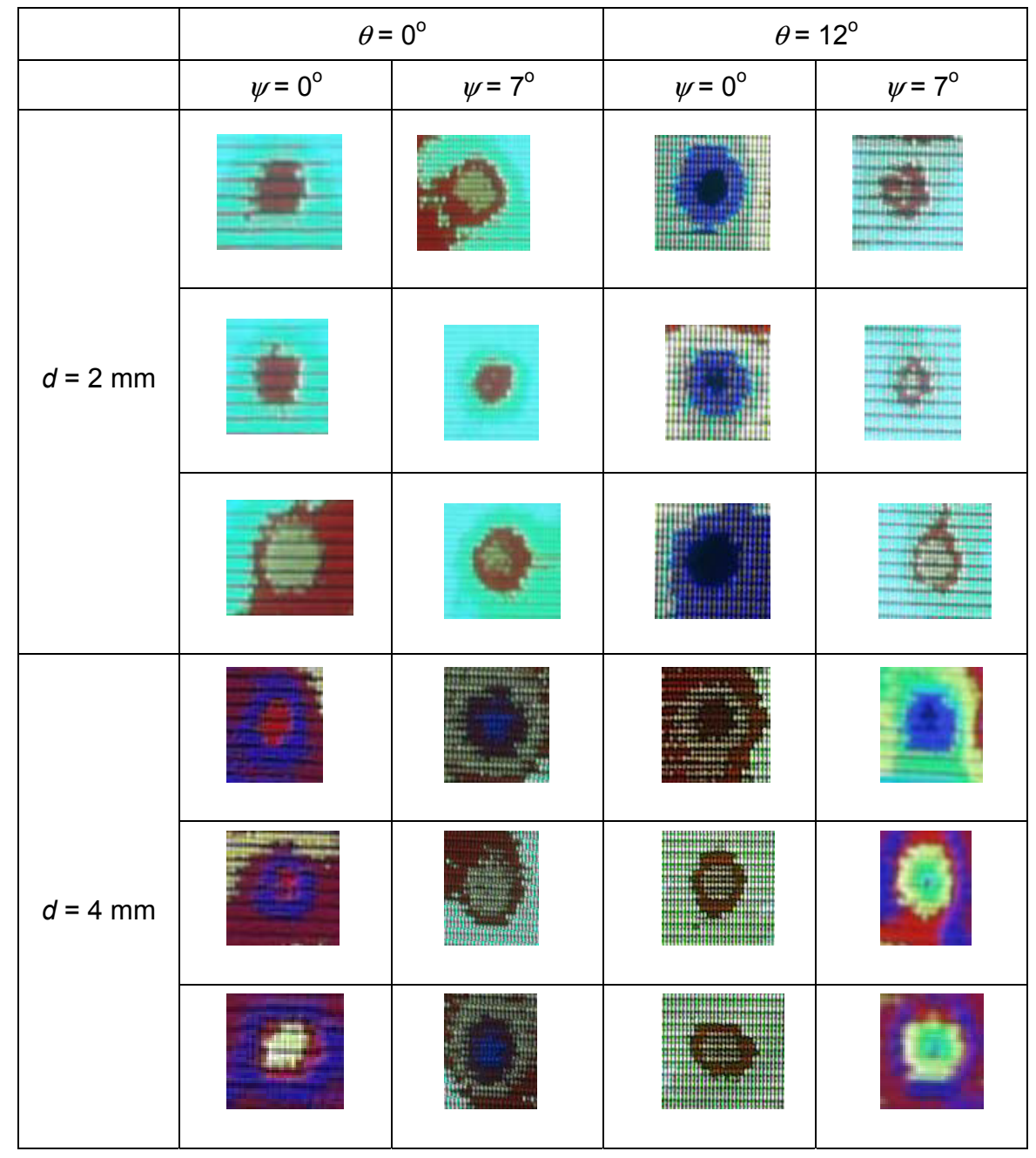

Fig. 4. Obtained thermograms 